\title{
ANALYTICAL APPROXIMATION \\ OF THE DISSIPATIVE STANDARD MAP
}

ALESSANDRA CELLETTI

Dipartimento di Matematica, Universitd di L'Aquila, I-67010 L'Aquila (Italy)

and

GABRIELLA DELLA PENNA and CLAUDE FROESCHLÉ

Observatoire de Nice, BP 229, F-06304 Nice Cedex 4 (France)

\section{Analytical Approximation}

We investigate the dynamics of a dissipative standard mapping defined by the equations

$$
\begin{aligned}
& y^{\prime}=y+\varepsilon \sin (x)-\alpha(y-\psi) \\
& x^{\prime}=x+y^{\prime}
\end{aligned}
$$

where $y \in \mathbf{R}, x \in \mathbf{T}$ and $\varepsilon$ is a real parameter; we refer to $0<\alpha<1$ as the "dissipative parameter" and to $\psi$ as the "dissipative coefficient" $(\varepsilon=\alpha=0$ provides an integrable mapping). Notice that the dynamics is contractive, since the jacobian of the above mapping equals to $1-\alpha$. In particular, we want to compare (see Celletti et al., 1997) the solutions associated to the conservative map (i.e., $\alpha=0$ ) with that related to (1) $(\alpha \neq 0)$. For simplicity, we consider the case when $\alpha=\varepsilon^{2}$ and construct explicit approximate solutions to the conservative and dissipative systems, using a suitable parametrization like in (Celletti and Chierchia, 1988).

In particular, we consider the curves described by the parametric equations

$$
\begin{aligned}
& x=\theta+u(\theta ; \varepsilon)+\alpha D w(\theta ; \varepsilon) \\
& y=\omega+u(\theta ; \varepsilon)-u(\theta-\omega ; \varepsilon)+\alpha[D w(\theta ; \varepsilon)-D w(\theta-\omega ; \varepsilon)]
\end{aligned}
$$

$(\theta \in \mathbf{T})$ where $u(\theta ; \varepsilon)$ and $w(\theta ; \varepsilon)$ are suitable functions. Here $D$ is the operator acting on a function $v(\theta ; \varepsilon)$ as

$$
D v(\theta ; \varepsilon)=v\left(\theta+\frac{\omega}{2} ; \varepsilon\right)-v\left(\theta-\frac{\omega}{2} ; \varepsilon\right) .
$$

Inserting (2) in (1) one gets

$$
\begin{array}{rl}
D^{2} & u(\theta ; \varepsilon)+\alpha D^{3} w(\theta ; \varepsilon)=\varepsilon \sin [\theta+u(\theta ; \varepsilon)+\alpha D w(\theta ; \varepsilon)] \\
& -\alpha(\omega-\psi+[u(\theta ; \varepsilon)-u(\theta-\omega ; \varepsilon)]+\alpha[D w(\theta ; \varepsilon)-D w(\theta-\omega ; \varepsilon)]) .
\end{array}
$$


Using analyticity properties, we expand $u, w$ and $\psi$ in Taylor series around $\varepsilon=0$ as

$$
u(\theta ; \varepsilon)=\sum_{j=0}^{\infty} u_{j}(\theta) \varepsilon^{j}, \quad w(\theta ; \varepsilon)=\sum_{j=0}^{\infty} w_{j}(\theta) \varepsilon^{j}, \quad \psi=\psi(\varepsilon)=\sum_{j=0}^{\infty} \psi_{j} \varepsilon^{j} ;
$$

inserting the above expansions in (3) and equating same orders of $\varepsilon$ one obtains explicit formulae for the functions $u_{j}, w_{j}$ and $\psi_{j}$. A finite truncation of the series expansions (4) provides an approximate solution of the equations of motion (see Celletti et al., 1997, for more details).

\section{Results}

We analyzed the behaviour of conservative invariant curves with irrational frequency $\omega$ and the corresponding dissipative solutions as $\varepsilon$ is varied. The approximate solutions look similar for low values of $\varepsilon$. In most cases the dynamics showed marked differences as $\varepsilon$ approaches the critical break-down value $\varepsilon_{c}$, due to analyticity properties as $\varepsilon<\varepsilon_{c}$ which are lost as $\varepsilon$ approaches the critical break-down threshold. Few exceptions were found in which conservative and dissipative dynamics are different for values much bigger than $\varepsilon_{c}$. A higher order solution should be computed in order to investigate these peculiar cases. We report in Table 1 the values $\varepsilon_{c}(\omega)$ representing the critical break-down threshold of the invariant curve with frequency $\omega$ and the values $\varepsilon_{C D}(\omega)$ at which the conservative and dissipative curves become different. In most cases these values may be thought as indicators of the onset of chaos. To provide some results, we report the values for the following rotation numbers: $\beta=\frac{\sqrt{5}-1}{2}, \omega_{1}=\frac{1}{2}+\frac{1}{100+\beta}, \omega_{2}=\frac{2}{3}+\frac{1}{20+\beta}, \omega_{3}=1-\frac{1}{10+\beta}$, $\omega_{4}=1-\frac{1}{100+\beta}$.

\section{TABLE I}

\begin{tabular}{|c|c|c|}
\hline Rotation number $\omega$ & $\varepsilon_{c}(\omega)$ & $\varepsilon_{C D}(\omega)$ \\
\hline$\beta$ & 0.971 & 0.32 \\
$\omega_{1}$ & 0.3 & 0.28 \\
$\omega_{2}$ & 0.7 & 0.5 \\
$\omega_{3}$ & 0.4 & 0.4 \\
$\omega_{4}$ & 0.05 & 0.5 \\
\hline
\end{tabular}

\section{References}

Celletti A., Chierchia L.: 1988, Construction of analytic KAM surfaces and effective stability bounds, Commun. in Math. Physics, 118, 119

Celletti A., Della Penna G., Froeschlé C.: 1997, Analytic approximation of the solution of the dissipative standard map, International Journal of Bifurcation and Chaos, in press. 